\title{
VideoMOOC-PL: Uma Linguagem de Padrões de Design Educacional para Apoiar a Produção de REAs na Forma de Vídeos para o Contexto dos MOOCs
}

\author{
Marcelo Fassbinder $^{1}$, Aracele G. O. Fassbinder ${ }^{2}$, Ellen F. Barbosa ${ }^{1}$ \\ ${ }^{1}$ Instituto de Matemática e Ciência da Computação (ICMC), Universidade de São Paulo \\ (USP), São Carlos, São Paulo, Brasil. \\ ${ }^{2}$ Instituto Federal de Educação, Ciência e Tecnologia do Sul de Minas Gerais \\ (IFSULDEMINAS), Muzambinho, Minas Gerais, Brasil. \\ marcello.fassbinder@usp.br, aracele.garcia@muz.ifsuldeminas.edu.br, \\ francinedicmc.usp.br
}

\begin{abstract}
Videos for Massive Open Online Courses (MOOCs) are usually produced using empirical knowledge or experiences from face-to-face courses or even traditional virtual courses. Thus, most of the videos produced do not have the desirable characteristics for courses in this format. So, this paper discusses the theoretical and practical aspects of development and evaluation of VideoMOOC-PL - an Educational Design Patterns Language that supports the production of Open Educational Resources (OER) in videos format, for the context of MOOCs. The results show that the videos produced using VideoMOOC-PL have more desirable characteristics for OER in the form of videos for the context of MOOCs than ad hoc decisions.
\end{abstract}

Resumo. Videos para Cursos Online Abertos e Massivos (MOOC) são geralmente produzidos utilizando conhecimento empírico ou experiências com o ensino presencial ou virtual formal. Dessa forma, grande parte dos vídeos produzidos não apresenta características desejáveis para cursos nesse formato. Sendo assim, este artigo discute os aspectos teóricos e práticos do desenvolvimento e avaliação da VideoMOOC-PL - uma Linguagem de Padrões de Design Educacional que apoia a produção de Recursos Educacionais Abertos (REA) na forma de vídeo, para o contexto dos MOOCs. Os resultados mostram que os vídeos produzidos utilizando a VideoMOOC-PL apresentam características mais desejáveis para REAs na forma de vídeos para o contexto dos MOOCs do que decisões ad hoc.

\section{Contexto}

Assistir a vídeos é provavelmente uma das atividades mais importantes no contexto virtual, aberto e massivo. Desse modo, compreender como os estudantes interagem com esse tipo de mídia é fundamental para melhorar a qualidade dos Massive Open Online Courses (MOOCs). Ações de clique, tais como pausar, pular e repetir, podem refletir a dificuldade do aprendiz em compreender o conteúdo do vídeo e indicar possíveis desistências do curso. Adicionalmente, Guo, Kim e Rubin (2014), Li et al. (2015) e Santos et al. (2015) destacam que vídeos em MOOCs merecem atenção especial, seja em questões pedagógicas, tecnológicas ou de design, pois, em geral, são criados por educadores com base em conhecimento empírico ou a partir de suas experiências na 
construção de cursos presenciais ou virtuais formais. Mas vídeos para MOOCs necessitam ser produzidos por meio de abordagens que considerem as especificidades para cursos nesse formato, já que nos MOOCs normalmente não existe o acompanhamento de um professor ou instrutor para esclarecer possíveis dúvidas dos estudantes.

Além disso, como os MOOCs fazem parte do movimento de Educação Aberta, bem como os Recursos Educacionais Abertos (REA), também é importante refletir sobre a produção dos vídeos considerando as diretrizes para construção dos REAs.

Essa situação desafiadora tem estimulado o desenvolvimento de abordagens baseadas em padrões para apoiar educadores ao projetar experiências inovadoras de aprendizagem no design de vídeos para MOOCs (Mor e Warburton 2016, Fassbinder 2018). Contudo, tais abordagens não contemplam todo o processo de design educacional de vídeos para MOOCs.

Padrões e Linguagens de Padrões (LPs) têm recebido muita atenção em diferentes áreas por se tratar de uma maneira simples para descrever e compartilhar conhecimento tácito e apoiar o design de novos produtos. A ideia de Padrões e LPs, como conhecida e utilizada hoje em dia, foi primeiramente definida pelo arquiteto Christopher Alexander, que reconheceu que um conjunto recorrente de problemas é encontrado sempre que um edifício é projetado (Pressman, 2005). Tais problemas recorrentes e suas soluções são caracterizados como padrões. De acordo com Alexander et al. (1977), cada padrão descreve um problema que ocorre repetidamente em nosso ambiente e, em seguida, descreve o núcleo da solução para esse problema de tal forma que qualquer pessoa pode usar a solução várias vezes. Já as linguagens de padrões são um conjunto de padrões agrupados e relacionados de uma forma a guiar o desenvolvimento de um produto (Alexander et al. 1977).

Nessa perspectiva, este artigo tem como objetivo investigar como uma linguagem de padrões de design educacional e seus padrões relacionados podem ser usados como uma maneira eficaz para apoiar equipes de MOOCs na produção de REAs na forma de vídeos.

O artigo está organizado da seguinte forma: a Seção 2 fornece uma visão geral do processo de desenvolvimento da LP. Na Seção 3 é apresentada a VideoMOOC-PL. O processo de avaliação da linguagem é apresentado na Seção 4. A Seção 5 apresenta as conclusões e perspectivas de trabalhos futuros.

\section{Processo de Criação da VideoMOOC-PL}

Para a criação da VideoMOOC-PL foi utilizada uma adaptação das diretrizes sugeridas por Meszaros e Doble (1997), Braga et al. (2007) e Iba, Sakamoto e Miyake (2011). O processo está dividido em seis etapas, aplicadas em um fluxo de forma interativa e incremental.

\subsection{Criação do Modelo de Domínio}

Segundo Braga et al. (2007), para criar uma LP que considere, de forma abrangente, aplicações em um determinado contexto, é necessário observar e coletar soluções que são comumente empregadas para solucionar problemas recorrentes em um determinado domínio. Assim, o ponto de partida para a criação da LP é a obtenção de um modelo para 
o domínio de destino, ou seja, um modelo que capture as atividades presentes na maioria das aplicações neste domínio.

Dessa forma, para a criação do modelo de domínio para produção de REAs na forma de vídeo para o contexto dos MOOCs foram investigados, inicialmente, os atos de especialistas na comunidade e identificadas as principais atividades comumente executadas na produção de conteúdos educacionais na forma de vídeos. Tais atividades foram primeiramente identificadas a partir de cinco fontes principais: (i) mapeamento sistemático para verificar quais estratégias para produção de vídeos educacionais vêm sendo utilizadas pelas equipes de MOOCs (Fassbinder et al., 2018); (ii) revisão de modelos e diretrizes para produção de REAs; (iii) estudo sobre a produção de vídeos educacionais; (iv) diretrizes para produção de vídeos compartilhadas pelos provedores de MOOCs; e (v) experiência dos autores na produção de vídeos educacionais para MOOCs. Depois de identificar as atividades, o próximo passo foi organizá-las. Para isso, foi definido um modelo chamado Ciclo de Vida para a Produção de REAs na Forma de Vídeo, apresentado na Subseção 2.1.1, que captura as atividades apresentadas na maioria dos processos de produção de REAs e vídeos educacionais analisados. $\mathrm{O}$ ciclo de vida também resume um conjunto de etapas da produção de REAs na forma de vídeo que podem ser aplicadas sistematicamente, com o objetivo de garantir qualidade aos vídeos produzidos.

\subsubsection{Ciclo de vida para produção REA na forma de vídeo}

Segundo Zancanaro (2015), durante o desenvolvimento de um REA é necessário que as etapas de produção sejam definidas de forma clara. Sendo assim, com o objetivo de avançar na exploração de uma estrutura comum de apoio à construção de REAs foi proposto um ciclo de vida para produção de REA na forma de vídeo. O ciclo de vida proposto foi elaborado a partir da junção dos principais modelos e ciclos de vida para produção de REA encontrados na literatura e apresentados na Tabela 1.

Tabela 1. Síntese dos Modelos e Ciclos para Produção de REAs

\begin{tabular}{|l|l|l|l|l|l|l|}
\hline $\begin{array}{c}\text { ADDIE } \\
\text { (Branch 2010; } \\
\text { Molenda 2003) }\end{array}$ & \multicolumn{1}{|c|}{$\begin{array}{c}\text { Gurell and } \\
\text { Wiley (2008) }\end{array}$} & \multicolumn{1}{|c|}{$\begin{array}{c}\text { Glahn et al. } \\
\mathbf{( 2 0 1 0 )}\end{array}$} & $\begin{array}{c}\text { Carrión et al. } \\
\mathbf{( 2 0 1 0 )}\end{array}$ & $\begin{array}{c}\text { Clements e } \\
\text { Pawlowski } \\
\mathbf{( 2 0 1 2 )}\end{array}$ & $\begin{array}{c}\text { Zancanaro } \\
\mathbf{( 2 0 1 5 )}\end{array}$ & $\begin{array}{c}\text { Arimoto } \\
\mathbf{( 2 0 1 6 )}\end{array}$ \\
\hline Analisar & Buscar & Criar & Analisar & Buscar & $\begin{array}{l}\text { Analisar } \\
\text { design }\end{array}$ & Planejar \\
\hline Design & Composição & Publicar & Design & Avaliar & Codificar & Design \\
\hline Desenvolver & Adaptar & $\begin{array}{l}\text { Localizar e } \\
\text { acessar }\end{array}$ & Desenvolver & Adaptar & Usar e avaliar & Desenvolver \\
\hline Implementar & Usar & $\begin{array}{l}\text { Enriquecimento } \\
\text { com Metadados }\end{array}$ & Implementar & Usar & $\begin{array}{l}\text { Abordagem } \\
\text { colaborativa }\end{array}$ \\
\hline Avaliar & Avaliar & & Compartilhar & Compartilhar & Avaliar \\
\hline
\end{tabular}

E, na sequência, a incorporação de um processo para produção de vídeos, que possui basicamente três etapas (Kindem; Musburger, 1997, Paolo et al., 2017):

- Pré-produção: Consiste no planejamento e preparação dos materiais para produzir o vídeo.

- Produção: Esta é a etapa onde são feitas as filmagens das cenas que compõem o vídeo.

- Pós-Produção: Nesta etapa ocorre a montagem das imagens, inserção da trilha sonora e dos efeitos do vídeo. 
O ciclo de vida proposto é apresentado na Figura 1 e está dividido em três fases relacionadas ao processo de produção de vídeo (pré-produção, produção e pós-produção). Dentro de cada uma dessas fases foram incorporadas as etapas do processo de produção de REA (análise, design, desenvolvimento, avaliação e distribuição), que são descritas a seguir.

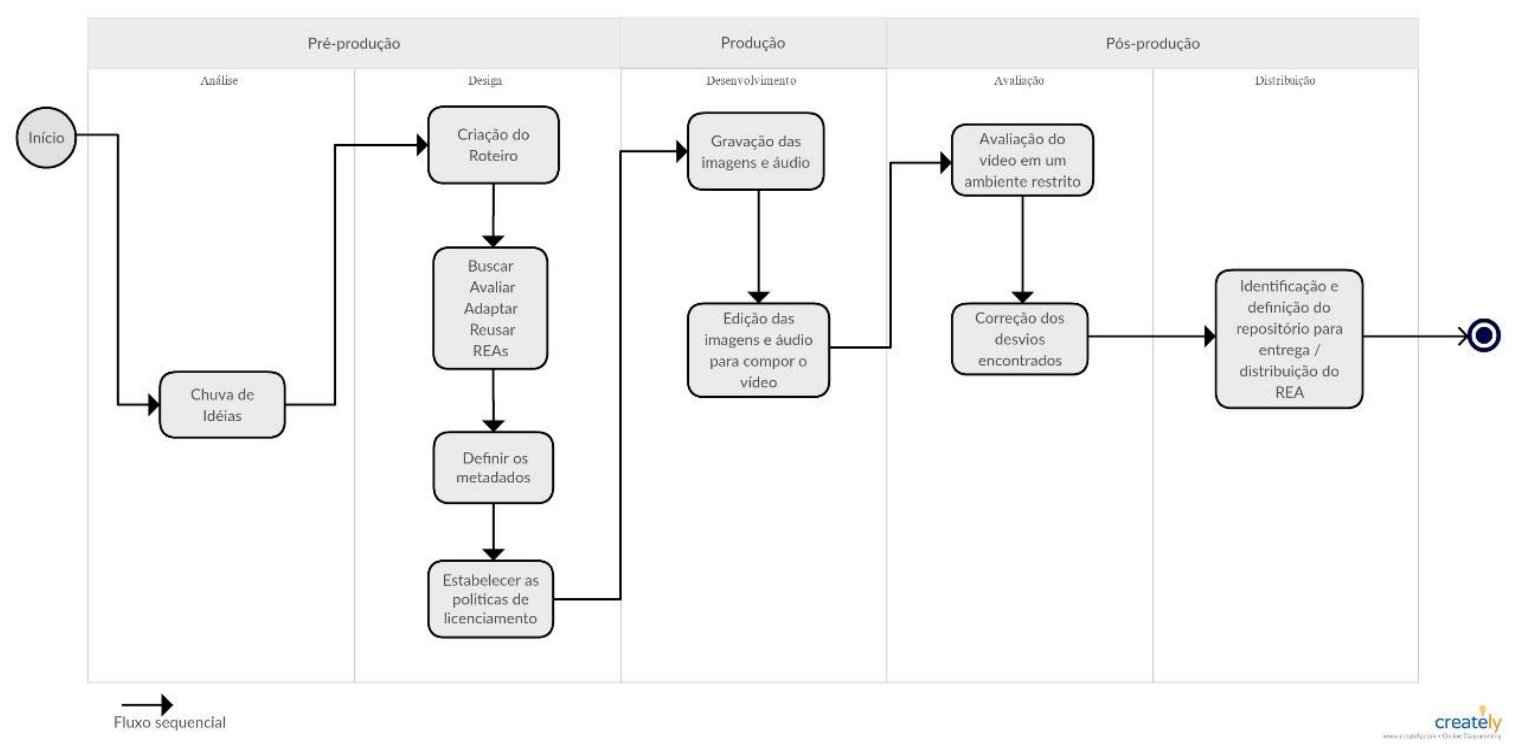

Figura 1. Ciclo de Vida para Produção de REAs na forma de Vídeos

- Análise: Nesta fase são discutidos os objetivos de aprendizagem, linguagem audiovisual e os recursos necessários para a produção do REA na forma de vídeo. Algumas das questões que necessitam ser respondidas nesta fase são: Quais são os objetivos de aprendizagem a serem atingidos com a produção do vídeo? Como aplicar a linguagem audiovisual para atender os objetivos de aprendizagem do vídeo? Existem recursos financeiros, humanos e tecnológicos suficientes para a produção do vídeo?

- Design: Esta fase determina como o objetivo de aprendizagem será alcançado. É fundamental estar atento aos detalhes nesta fase. O foco deve estar nos objetivos de aprendizagem para produção do roteiro, na busca, avaliação e reutilização de REA, na definição dos metadados e na utilização de licenças preferencialmente abertas.

- Desenvolvimento: Nesta fase acontece a captura das imagens e áudios, e a composição do vídeo. Todo o processo deve seguir o que foi proposto na fase de design utilizando o roteiro como artefato para orientar toda a equipe envolvida.

- Avaliação: Ao final da etapa de desenvolvimento, toda a equipe deve se reunir para discutir, avaliar e aprovar/desaprovar o vídeo produzido. Um conjunto de atividades deve ser conduzido para avaliar e aprovar o REA.

- Distribuição: O REA na forma de vídeo deve ser disponibilizado em um ambiente apropriado para sua utilização, o que é fundamental para identificar pontos fracos e propor melhorias. O acesso efetivo ao vídeo deve ser feito por meio de plataformas, repositórios e sites institucionais ou autônomos, incluindo metadados e licenças associadas, com pouca ou nenhuma restrição à sua (re)utilização. A ampla disponibilidade do vídeo permite que outros usuários 
possam utilizá-lo e adaptá-lo ao seu próprio contexto e necessidades.

\subsection{Mineração de Padrões}

O modelo resultante da etapa anterior ajuda a identificar as atividades realizadas recorrentemente pelos especialistas e, em seguida, a entender as formas para realizar tais atividades. Segundo Iba, Sakamoto e Miyake (2011), os conhecimentos extraídos são ideias potenciais para padrões, também chamados de padrões candidatos (exemplos de padrões candidatos: briefing, roteiro, etc.). Os padrões candidatos foram agrupados em "Famílias de Padrões", que compartilhavam certos objetivos de design educacional de vídeos para MOOCs. As "Famílias de Padrões" representam as principais fases do ciclo de vida para produção de REAs na forma de vídeo (análise, design, desenvolvimento, avaliação e distribuição).

\subsection{Padrões Determinados}

O ciclo de vida para produção de REAs na forma de vídeo e o conjunto de padrões candidatos foram utilizados para identificar mais padrões relacionados ao contexto de design educacional de vídeos para MOOCs, refinar os atuais com características desejáveis de vídeos para cursos no formato $\mathrm{MOOC}$, e guiar a criação do gráfico da VideoMOOC-PL.

\subsection{Escrita dos Padrões}

Para a escrita dos padrões utilizou-se uma adaptação dos formatos definidos por Iba, Sakamoto e Miyake (2011) e Meszaros e Doble (1997). Dessa forma, os padrões foram escritos e detalhados utilizando a seguinte estrutura:

- nome curto e memorável que descreve o padrão facilmente;

- uma linha que explica o significado do padrão;

- ilustração;

- contexto no qual o padrão pode ser usado;

- problema que pode ocorrer no contexto descrito;

- as forças são aspectos que podem deixar o problema difícil de ser resolvido;

- a solução para o problema é breve e abstratamente apresentada;

- as ações descrevem a solução, de uma forma mais concreta e detalhada.

\subsection{Criação do gráfico da VideoMOOC-PL}

A maneira como os padrões se relacionam dentro da linguagem deve estar claramente especificada. Portanto, foi criado um gráfico que mostra a interação dos padrões e seu fluxo de aplicação. O gráfico da VideoMOOC-PL é apresentado na Figura 2.

\subsection{Avaliação da linguagem de padrões}

Algumas estratégias foram utilizadas para avaliar o ciclo de vida para a produção de REAs na forma de vídeo e, principalmente, a VideoMOOC-PL. O processo de avaliação é descrito com mais detalhes na Seção 4. 


\section{Visão Geral da VideoMOOC-PL}

A VideoMOOC-PL é uma linguagem de padrões de design educacional que visa auxiliar as equipes durante o processo de produção de vídeos educacionais para serem utilizados em cursos do tipo MOOC. A linguagem também visa incentivar a criação de vídeos para serem disponibilizados como REAs, contribuindo para a disseminação do conhecimento e ampliando o acesso à educação de qualidade. A Figura 2 fornece uma visão geral da VideoMOOC-PL.

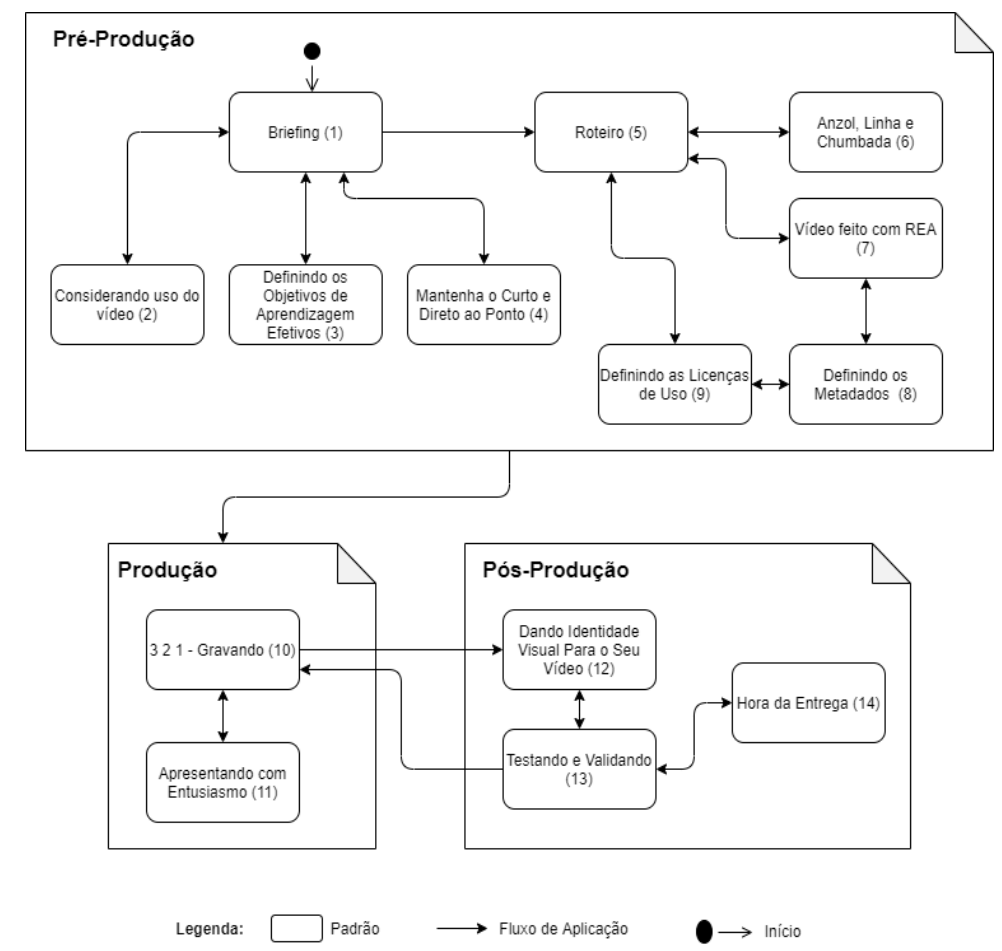

Figura 2. VideoMOOC-PL: Gráfico com Fluxo de Aplicação dos 14 Padrões que compõem a Linguagem

No total, 14 padrões foram identificados e agrupados em três categorias relacionadas às etapas de produção de vídeos: pré-produção, produção e pós-produção. A título de ilustração, a seguir é apresentado o padrão Mantenha-o Curto e Direto ao Ponto, relacionado à fase de pré-produção. Os demais padrões podem ser acessados a partir do site https://sites.google.com/view/video-pl.

\section{Mantenha-o Curto e Direto ao Ponto}

Vídeos curtos, independentes, mas que se relacionam.

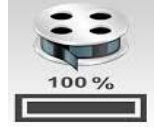

Você está produzindo material didático para o seu MOOC, entregue em formato de vídeo. Isso inclui todo tipo de conteúdo abordado no MOOC, o qual os alunos devem seguir para atingir os objetivos de aprendizagem. 
IX Congresso Brasileiro de Informática na Educação (CBIE 2020)

Anais do XXXI Simpósio Brasileiro de Informática na Educação (SBIE 2020)

\section{Problema}

Muitos cursos online são baseados na entrega de conteúdo que segue uma abordagem similar a das aulas presenciais. Mas você sabe que a criação de um vídeo completo com um conteúdo extenso para um curso MOOC é muito cansativo, tanto do ponto de vista do ensino quanto do aprendizado do aluno. Você não conseguirá manter seu entusiasmo na apresentação em um vídeo longo e sabe que a atenção do aluno cairá drasticamente em vários pontos em uma única gravação longa.

\section{Forças}

É difícil criar vídeos que prendam a atenção do estudante por muito tempo.

Em aulas expositivas em ambientes presenciais, o professor pode utilizar diversos recursos para manter a atenção dos estudantes.

\section{Solução}

Portanto, crie vídeos de conteúdos específicos, dividindo conteúdos muito extensos em pequenos tópicos, nos quais você possa se sentir confiante, sendo assistido por seus alunos na íntegra. Certifique-se de que o material seja projetado dentro de uma estrutura que permita seu reuso e adaptação em diferentes contextos.

\section{Ações}

Não há regras rígidas e rápidas em relação à duração exata de um vídeo. No entanto a maneira como os espectadores se envolvem com o vídeo educacional online tende a ser diferente da televisão ou do cinema. Como as taxas de abandono dos espectadores também são muito mais altas para vídeos online do que outras mídias, apresentar os conceitos no menor tempo possível é essencial. Portanto, os conceitos mais importantes devem ser apresentados primeiro, se possível. Quando há a necessidade de transmitir vários conceitos, tente estruturar esse conteúdo em torno de vários vídeos mais curtos. Vídeos mais curtos permitem um potencial de auto direção no aprendizado, proporcionando aos alunos opções sobre o que assistir e quando assistir, requisito muito importante para os MOOCs.

\section{Avaliação}

Esta seção resume uma combinação de métodos quantitativos e qualitativos usados para validar a VideoMOOC-PL. De acordo com Goodyear et al. (2004), o desenvolvimento, avaliação e disseminação de padrões de design e de linguagens associadas são atividades colaborativas e geralmente se estendem por vários anos. Além disso, Braga et al. (2007) destacam que uma avaliação completa de uma LP é uma tarefa difícil, pois muitas aplicações são necessárias para garantir sua utilidade. Sendo assim, para apoiar a fase de avaliação, tais autores sugerem o uso de ferramentas online, como wikis e conferências, por exemplo a Pattern Languages of Programs (PLoP) ${ }^{1}$, que passam por rigorosas "estratégias de pastoreio" antes e durante a realização do evento em si.

Nessa perspectiva, as avaliações da VideoMOOC-PL foram organizadas em três grupos principais: (i) avaliação por especialistas em padrões; (ii) estudo de campo; e (iii) satisfação dos usuários que interagiram com a linguagem. Desse modo, a VideoMOOCPL foi avaliada por meio de uma avaliação por especialistas, que serviu como método de validação interna. Além disso, foi conduzida uma avaliação em campo, considerando um estudo de caso com 22 alunos de um curso de Ciência da Computação, em que a VideoMOOC-PL foi utilizada como um guia para projetar REAs na forma de vídeos para MOOCs, e uma avaliação de satisfação com os mesmos alunos que interagiram com a linguagem. Ambas avaliações foram consideradas como um método de avaliação externa.

\section{Avaliação por Especialistas}

As conferências PLoP oferecem processo de revisão especial e apoio aos autores que

\footnotetext{
${ }^{1}$ https://www.hillside.net/plop/2020/
} 
desejam receber feedback de seus padrões por colegas autores com experiência na escrita de padrões. A VídeoMOOC-PL e seus padrões foram refinados por meio de um longo processo de pastoreio ${ }^{2}$ na conferência PLOP $^{3}$ (2019), organizada pela comunidade Hillside $^{4}$ (Fassbinder et al., 2019). O feedback dos especialistas serviu para refinar a escrita dos padrões já identificados e extrair novos padrões.

\section{Avaliação de Campo}

Para esta avaliação, foi conduzido um estudo de campo com estudantes do curso de Ciência da Computação, do IFSULDEMINAS campus Muzambinho, onde os alunos deveriam pesquisar, compreender e criar conteúdo na forma de vídeo para 10 estratégias ativas de ensino-aprendizagem (Gallery Walk, OPERA, Think-Pair-Share, entre outras). Tais conteúdos seriam utilizados na criação e oferta de um MOOC sobre estratégias ativas. Para isso os estudantes foram divididos em cinco grupos, onde cada grupo ficou responsável por duas estratégias ativas. Cada grupo deveria produzir um vídeo utilizando abordagem ad hoc, e outro vídeo sobre a segunda estratégia, mas dessa vez utilizando a VideoMOOC-PL como um guia para produzir o vídeo. Para essa avaliação participaram 22 estudantes, sendo um total de 5 vídeos produzidos de forma ad hoc e 5 vídeos produzidos utilizando a VideoMOOC-PL. Para descrever o objetivo de pesquisa foi utilizada a estrutura Objetivo/Pergunta/Métrica (GQM) (Van Solingen e Berghout 1999). Também foram utilizados questionários específicos para investigar o nível de satisfação, confiança, facilidade de uso, e intenção dos sujeitos em utilizar a linguagem para criar vídeos.

Uma rubrica específica para validar os vídeos foi usada para medir os efeitos dos métodos (ad hoc versus VideoMOOC-PL). A rubrica utilizada para avaliar os vídeos tem um total de cinco dimensões de características desejáveis para a criação de REAs na forma de vídeos, onde cada dimensão contém vários itens. Para a avaliação, os estudantes verificaram até que ponto os vídeos produzidos contemplavam esses itens. $\mathrm{Na}$ Figura 3 são apresentados os resultados relacionados à dimensão de "análise", que contém sete itens, analisados em 10 vídeos. As bolas representam a média das respostas referentes aos sete itens da dimensão "análise" para cada vídeo produzido. Os valores utilizados seguem a escala

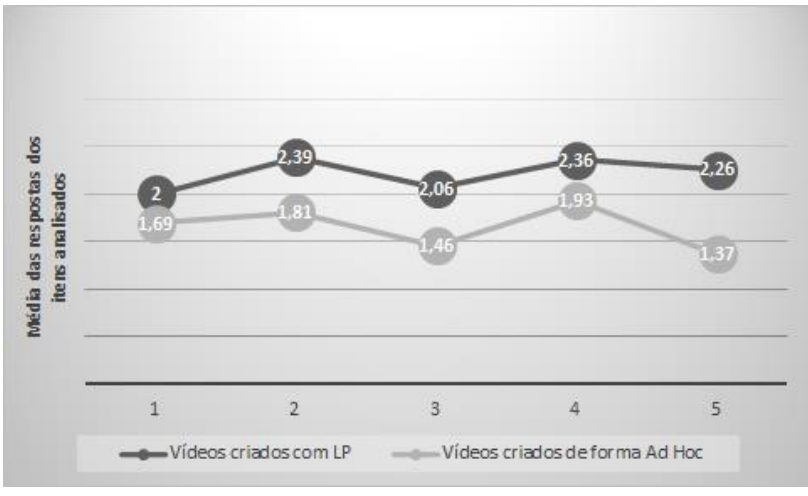

Figura 3. Resultado das Avaliação dos Vídeos produzidos utilizando a Abordagem Ad Hoc versus a VideoMOOC-PL

Likert de quatro pontos ([0] Não contemplado; [0.1-1.0] Parcialmente contemplado; [1.12.0] Suficientemente contemplado; [2.1-3.0] Totalmente contemplado).

Em geral, a média dos valores pertencentes aos vídeos criados com o apoio da VideoMOOC-PL é maior que os vídeos criados utilizando a abordagem ad hoc. Essa

\footnotetext{
${ }^{2} \mathrm{http}$ //hillside.net/the-language-of-shepherding for more details on the process.

${ }^{3} \mathrm{https}$ //www.hillside.net/plop/2019/index.php?nav=PLoP19

${ }^{4}$ https://www.hillside.net
} 
tendência foi observada também nas outras quatro dimensões. Portanto, há evidências de que os vídeos criados com apoio da VideoMOOC-PL apresentam características mais desejáveis para REA na forma de vídeos para o contexto dos MOOCs do que aqueles criados a partir da abordagem ad hoc ou seguindo decisões aleatórias.

Também foram observadas boas evidências sobre o uso da VideoMOOC-PL considerando o nível de satisfação, confiança, facilidade de uso e a intenção de uso futuro, conforme resumido na Tabela 2. Onde os participantes relataram uma maior confiança de estarem produzindo o vídeo de maneira correta, além de ficarem mais satisfeitos com o resultado dos vídeos produzidos utilizando a VideoMOOC-PL como apoio.

Tabela 2. Resultado do Questionário aplicado para Verificar o Nível de Satisfação no Uso da VideoMOOC-PL

\begin{tabular}{|l|c|c|c|c|}
\hline & Concordo Fortemente & Concordo & Nem Concordo nem discordo & Discordo \\
\hline Satisfação quando usando a LP & $10 \%$ & $75 \%$ & $15 \%$ & - \\
\hline Nível de confiança. & $5 \%$ & $65 \%$ & $30 \%$ & - \\
\hline A LP é fácil de usar & $15 \%$ & $70 \%$ & $15 \%$ & - \\
\hline Intenção de uso futuro & $55 \%$ & $45 \%$ & - & - \\
\hline
\end{tabular}

\section{Conclusões e Trabalhos Futuros}

Neste artigo discutimos a pesquisa, desenvolvimento e avaliação de uma LP de design educacional para apoiar a produção de REAs na forma de vídeos para o contexto dos MOOCs. A VideoMOOC-PL contém um total de 14 padrões que estão agrupados em três categorias relacionadas às etapas de produção de vídeos educacionais. $\mathrm{O}$ artigo também apresenta um ciclo de vida para produção de REAs na forma de vídeos que apoia as principais etapas e atividades relacionadas à produção de REAs em vídeos.

A análise dos resultados preliminares da avaliação interna e externa indicaram que a VideoMOOC-PL pode ser efetivamente aplicada para orientar todo o processo de design de REAs na forma de vídeo para o contexto dos MOOCs. Como trabalhos futuros pretende-se aplicar uma avaliação com especialistas na produção vídeos para o contexto dos MOOCs, com objetivo de refinar e identificar novos padrões para compor a linguagem, além da oferta de oficinas e escrita de artigos divulgar a VideoMOOC-PL.

\section{Agradecimentos}

Os autores agradecem às agências de fomento brasileiras (CNPq, Fapemig, CAPES), e à FAPESP (Processo 2018/26636-2) pelo apoio financeiro.

\section{Referências}

Alexander, C.; Ishikawa, S.; Silverstein, M.; Jacobson, M.; Fiksdahl-king, I.; Angel, S. A pattern language: Towns, buildings, construction . Oxford University Press New York, 1977.

Braga, R. T. V., Ré, R. and Masiero, P. C. (2007) "A Process to Create Analysis Pattern Languages for Specific Domains", In: Proceedings of SugarLoafPLoP, 2007.

Branch, R. M. Instructional design: The ADDIE approach. [s.l: s.n.], 2010.

Fassbinder, M.; Fassbinder, A. G. O. and Barbosa, E. F. Construção de Vídeos para MOOCs: Um 
IX Congresso Brasileiro de Informática na Educação (CBIE 2020)

Anais do XXXI Simpósio Brasileiro de Informática na Educação (SBIE 2020)

Mapeamento Sistemático. In: XXIII Congresso Internacional de Tecnologia Educativa (TISE), Brasilia, Brasil, Novembro, 2018.

Fassbinder, M. ; Fassbinder, A. G. O. ; Fioravanti, M. L. ; Barbosa, E. F. . Towards an Educational Design Pattern Language to support the development of Open Educational Resources in videos for the MOOC context. In: 26th Conference on Pattern Languages of Programs (PLoP 2019), 2019, Ottawa (ON) - Canadá.

Fassbinder, A. G. O. A contribution to the process of designing for learning in Masive Open Online Courses (MOOCs): Tese (Doutorado) Universidade de São Paulo, Instituto de Ciências Matemáticas e de Computação, 2018.

Gamma, E., R. Helm, R. Johnson, and J. Vlissides. (1995) “Design Patterns: Elements of Reusable Object-Oriented Software".

Gurell, S.; Wiley, D. OER handbook for educators, version 1.0. 2008.

Goodyear, P., Avgeriou, P., Baggetun, R., Bartoluzzi, S., Retalis, S., Ronteltap, F. and Rusman, R. (2004) "Towards a pattern language for networked learning", Networked Learning Conference, pp. 449-455.

Guo, P. J.; Kim, J.; Rubin, R. How video production affects student engagement: an empirical study of mooc videos. In: ACM. Proceedings of the first ACM conference on Learning@scale conference. [S.1.], 2014. p. 41-50.

Iba, T., Sakamoto, M. and Miyake, T. (2011) "How to write tacit knowledge as a Pattern Language: Media Design for spontaneous and collaborative communities", Social and Behavioral Sciences 26, pp. 46-54.

Kindem, G. A., Musburger, R. B. Introduction to media production: from analog todigital. [S.1.]: Butterworth-Heinemann, 1997

Li, N.; Kidzin' Ski, L.; Jermann, P.; Dillenbourg, P. Mooc video interaction patterns: What do they tell us? In: Design for teaching and learning in a networked world. [S.1.]: Springer, 2015.

Meszaros G.; Doble, J. Metapatterns: A pattern language for pattern writing. In: Pattern languages of program design. [S.1.: s.n.], 1997. p. 1-36.

Mor, Y.; Warburton, S. L. Patterns for using video in moocs. EUROPLOP, 2016.

Molenda, M. In Search of the Elusive ADDIE Model. Performance Improvement, v. 42, n. 5, p. 34-36, 2003.

Paolo, T. D, Wakefield, J. S.; Mills, 1. A., Baker, L. Lights, camera, action: Facilitating the design and production of effective instructional videos. TechTrends, Springer, 2017.

Pressman, R. S. Software enginee-ring: a practitioner's approach. Palgrave Macmilan, 2005.

Santos, A. M.; Costa, F.; Viana, J.; Silva, A. G. Estratégias para desenho e produção de vídeos para cursos em formato MOOC. In: Actas da IX Conferência Internacional de Tecnologias de Informação e Comunicação na Educação, Challenges. 2015.

Schmidt, D.; Stal, M.; Rohnert, H.; Buschmann, F. Pattern-Oriented Software Architectu-re, Volume 1: A System of Patterns. [S.1.]: John Wiley \& Sons, 1996.

Van Solingen, R., Berghout, E. (1999) “The Goal/Question/Metric Method: A Practical Guide for Quality Improvement and Software Development", McGraw-Hill International, London/Chicago.

Zancanaro, A. Produção de recursos educacionais abertos com foco na disseminação do conhecimento: uma proposta de framework. 2015. 192 f. Centro Tecnológico, Universidade Federal de Santa Catarina, Florianópolis, 2015. 